\title{
Prospects for measuring coherent neutrino-nucleus elastic scattering at a stopped-pion neutrino source
}

\author{
Kate Scholberg ${ }^{1}$ \\ ${ }^{1}$ Department of Physics, Duke University, Durham, NC 27708 USA
}

(Dated: September 25, 2018)

\begin{abstract}
Rates of coherent neutrino-nucleus elastic scattering at a high-intensity stopped-pion neutrino source in various detector materials (relevant for novel low-threshold detectors) are calculated. Sensitivity of a coherent neutrino-nucleus elastic scattering experiment to new physics is also explored.

PACS numbers: 13.15.+g, 13.40.Em, 23.40.Bw
\end{abstract}

\section{INTRODUCTION}

Coherent elastic neutral current (NC) neutrinonucleus scattering 1, 2] has never been observed. In this process, a neutrino of any flavor scatters off a nucleus at low momentum transfer $Q$ such that the nucleon wavefunction amplitudes are in phase and add coherently. The cross section for a spin-zero nucleus, neglecting radiative corrections, is given by [3] ,

$$
\left(\frac{d \sigma}{d E}\right)_{\nu A}=\frac{G_{F}^{2}}{2 \pi} \frac{Q_{w}^{2}}{4} F^{2}(2 M E) M\left[2-\frac{M E}{k^{2}}\right]
$$

where $k$ is the incident neutrino energy, $E$ is the nuclear recoil energy, $M$ is the nuclear mass, $F$ is the ground state elastic form factor, $Q_{w}$ is the weak nuclear charge, and $G_{F}$ is the Fermi constant. The condition for coherence requires that $Q \lesssim \frac{1}{R}$, where $R$ is the nuclear radius. This condition is largely satisfied for neutrino energies up to $\sim 50 \mathrm{MeV}$ for medium $A$ nuclei 2, 4].

For neutrino energies up to $\sim 50 \mathrm{MeV}$, typical values of the total coherent elastic cross section are in the range $\sim 10^{-39} \mathrm{~cm}^{2}$, which is relatively high compared to other neutrino interactions in that energy range (e.g. charged current $(\mathrm{CC})$ inverse beta decay on protons has a cross section $\sigma_{\bar{\nu}_{e} p} \sim 10^{-40} \mathrm{~cm}^{2}$, and elastic neutrino-electron scattering has a cross section $\sigma_{\nu_{e} e} \sim 10^{-43} \mathrm{~cm}^{2}$ ).

In spite of its large cross section, coherent elastic neutrino-nucleus scattering has been difficult to observe due to the very small resulting nuclear recoil energies: the maximum recoil energy is $\sim 2 k^{2} / M$, which is in the sub-MeV range for $k \sim 50 \mathrm{MeV}$ for typical detector materials (carbon, oxygen). Such energies are below the detection thresholds of most conventional high-mass neutrino detectors. Although there have been suggestions to look for coherent elastic $\nu A$ scattering of reactor, spallation source, solar, supernova, or geophysical neutrinos [2, 5, 6, 7], as yet there has been no successful detection.
However, in recent years there has been a surge of progress in development of novel ultra low threshold detectors, many with the aim of weakly interacting massive particle recoil detection or very low energy solar neutrino detection. Thresholds of $10 \mathrm{keV}$ or even lower for detection of nuclear recoils may be possible. Such detectors include (but are not limited to): noble element (neon, argon, xenon) scintillation, ionization or tracking detectors, solid state detectors (germanium, silicon), bubble and superheated droplet detectors 5, 6, 8, 9, 10, 11, 12, 13, $14,15,16,17,18,19]$. Some of these new technologies (for instance noble liquid detectors such as CLEAN 8]) may plausibly attain ton-scale masses in the relatively near future.

A promising source of neutrinos for measurement of coherent elastic cross sections is that arising from a stopped-pion source. Monoenergetic $29.9 \mathrm{MeV} \nu_{\mu}$ 's are produced from pion decay at rest, $\pi^{+} \rightarrow \mu^{+} \nu_{\mu}$, and $\bar{\nu}_{\mu}$ and $\nu_{e}$ from $\mu^{+} \rightarrow \nu_{e} e^{+} \bar{\nu}_{\mu}$ follow on a muon-decay timescale $(\tau=2.2 \mu \mathrm{s})$. The neutrino spectral shape is shown in Fig. 11 Neutrinos in this energy range will produce nuclear recoils from coherent scattering with tens of $\mathrm{keV}$. If the beam is pulsed in a short $(<\mu \mathrm{s})$ time window, the pion decay $\nu_{\mu}$ will be prompt with the beam, and the muon-decay products will be delayed.

Stopped-pion sources of neutrinos have been employed for neutrino experiments in the past 20, 21], and future high-flux facilities are planned 22]. The Spallation Neutron Source (SNS) at Oak Ridge National Laboratory should turn on in 2006; J-PARC is another near-term possibility [23]. An experiment to measure neutrino-nucleus $\mathrm{CC}$ and NC cross sections (relevant for supernova physics and detection) in the tens of $\mathrm{MeV}$ range [24, 25] with conventional detectors at the SNS has already been proposed. A shielded concrete bunker is envisioned at a location $20 \mathrm{~m}$ from the source; this could potentially accommodate a low-threshold detector as well as the currently planned detectors. The SNS beam is pulsed, with less than microsecond width and a $60 \mathrm{~Hz}$ frequency. 


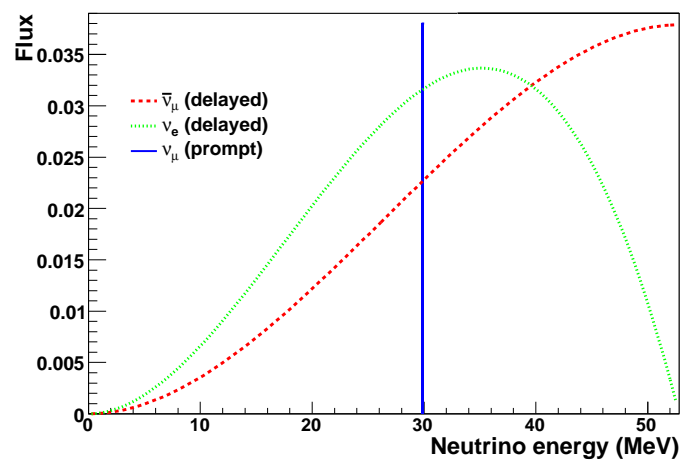

FIG. 1: Shape of neutrino spectra from a stopped-pion source, for the different produced flavors.

Here prospects for measuring coherent elastic neutrino-nucleus scattering will be evaluated using parameters relevant for the SNS; however the results should be generally applicable to experiments at any high-intensity stopped-pion $\nu$ source.

\section{EXPECTED EVENT RATES}

The expected rate of interactions differential in recoil energy is given by

$$
\frac{d N}{d E}=N_{t} \int d k \phi(k) \frac{d \sigma}{d E}(k),
$$

where $N_{t}$ is the number of targets and $\phi(k)$ is the incident neutrino flux. Spectra for $\nu_{\mu}, \bar{\nu}_{\mu}$ and $\nu_{e}$ for a stopped $\pi^{+} / \mu^{+}$source, assuming $\sim 10^{7} \nu \mathrm{s}^{-1}$ $\mathrm{cm}^{-2}$ per flavor at $20 \mathrm{~m}$ from the source are assumed. Cross sections and form factors from [3, 26] for ${ }^{20} \mathrm{Ne}$, ${ }^{40} \mathrm{Ar},{ }^{76} \mathrm{Ge}$, and ${ }^{132} \mathrm{Xe}$ are used. Figs. 2 through 5 show the results. The rates are quite promising: for a ton-scale detector with a few to $10 \mathrm{keV}$ threshold, $10^{4}-10^{5}$ signal events per year are expected. Even for kilogram-scale detectors, event rates may be in the tens per year.

Fig. 6]plots integrated yield over threshold for several elements for comparison. One can see that the higher the nuclear mass, the higher the overall event rate at low threshold (scaling approximately as the square of the number of neutrons), but the smaller the typical recoil energies $\left(E_{\max }=2 k^{2} / M\right)$.

In the absence of a specific detector model, perfect detection efficiency and zero background are assumed, which is not realistic. Detection efficiencies for many low-threshold detector types (see Sec. II) can be reasonably high, but can depend on background levels. Backgrounds will include beamrelated neutrons, cosmogenics, radioactivity and instrumental background, as well as other CC and NC
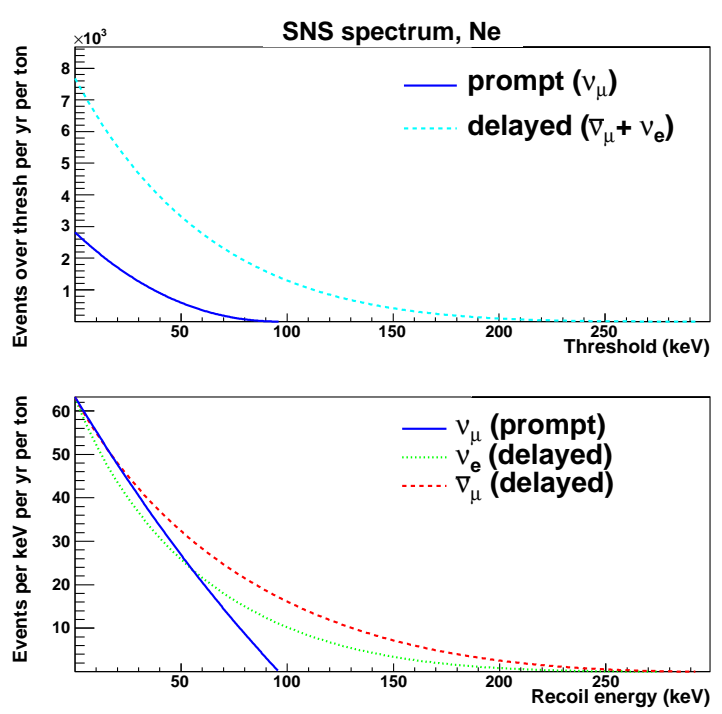

FIG. 2: Bottom panel: Differential yield at the SNS in 1 ton of ${ }^{20} \mathrm{Ne}$ (solid: $\nu_{\mu}$, dotted: $\nu_{e}$, dashed: $\bar{\nu}_{\mu}$ ) per year per $\mathrm{keV}$, as a function of recoil energy. Top panel: Number of interactions over recoil energy threshold in 1 ton of ${ }^{20} \mathrm{Ne}$ for $1 \mathrm{yr}$ of running at the SNS (solid: $\nu_{\mu}$, dashed: sum of $\nu_{e}$ and $\bar{\nu}_{\mu}$ ), as a function of recoil energy threshold.
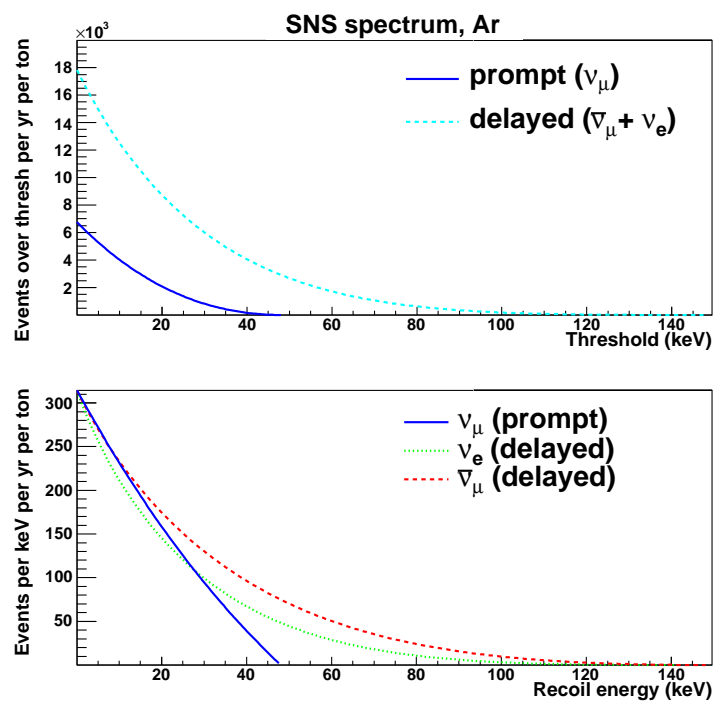

FIG. 3: As in Fig. 2 for ${ }^{40}$ Ar.

neutrino reactions; these will need to be evaluated for a specific detector's rejection capabilities and location. Backgrounds are not obviously overwhelming, especially given that the pulsed structure of the beam such as that at the SNS leads to a powerful rejection factor $\left(\sim 4 \times 10^{-4}\right)$ against steady-state backgrounds. It is not really clear at this point whether beam-related backgrounds will be worse for prompt or delayed neutrinos; it will depend on shielding and 

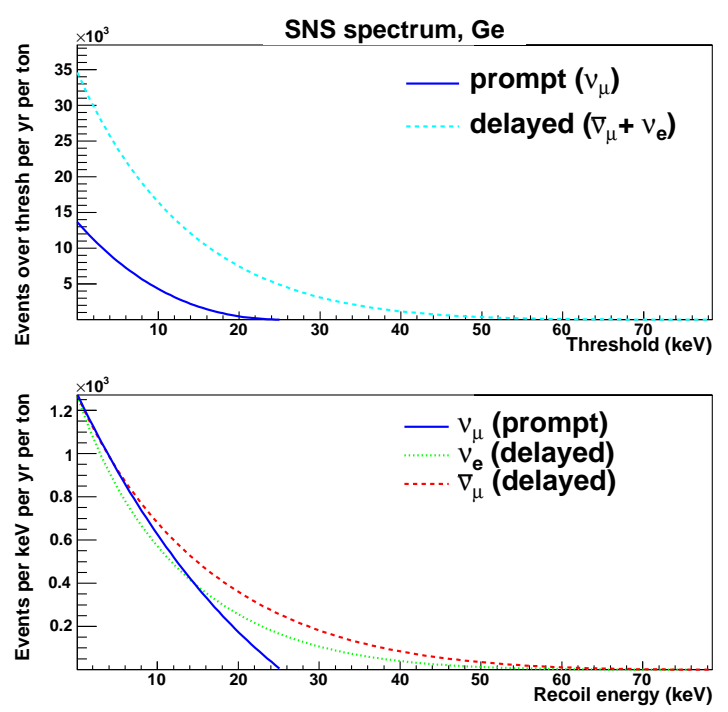

FIG. 4: As in Fig. 2 for ${ }^{76} \mathrm{Ge}$.
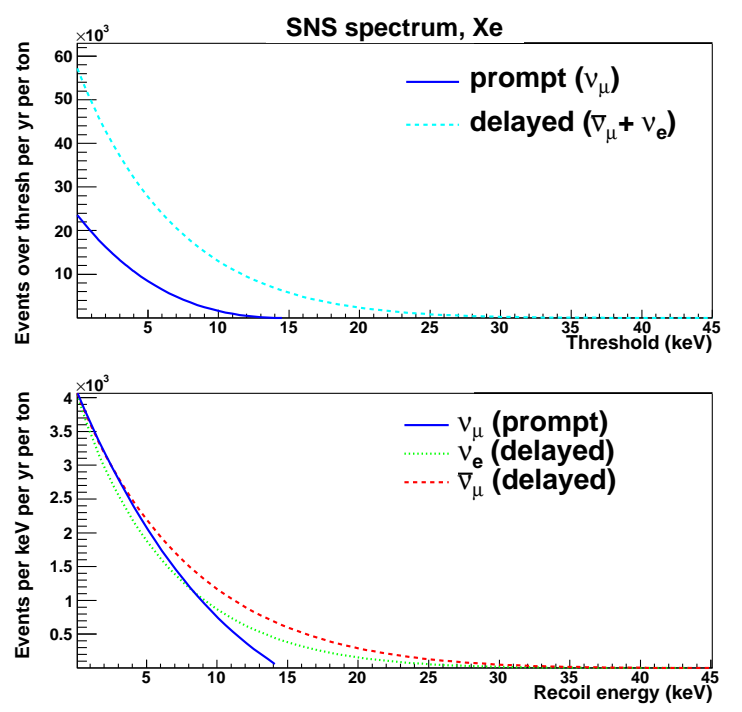

FIG. 5: As in Fig. 2 for ${ }^{132} \mathrm{Xe}$.

detector location. Therefore the contributions from prompt and delayed fluxes are given separately.

\section{PHYSICS POTENTIAL}

The neutrino-nucleus coherent elastic scattering cross section is cleanly predicted by the Standard Model (SM); form factors can be known to better than $5 \%$, and radiative corrections are known at the percent level 27]. Any measured deviations from prediction would be interesting [28]. In the context of the SM, the weak mixing angle is related to the absolute scattering rate. One can also constrain
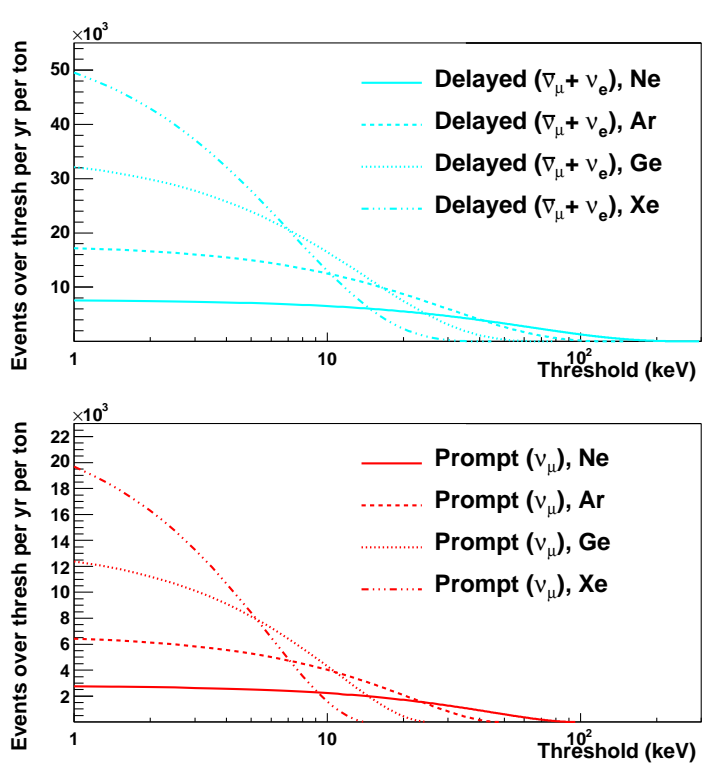

FIG. 6: The number of interactions over the recoil energy threshold for various detector materials (bottom panel: prompt $\nu_{\mu}$, top panel: sum of delayed $\nu_{e}$ and $\bar{\nu}_{\mu}$ ).

non-standard interactions (NSI) of neutrinos and nucleons. Also, non-zero neutrino magnetic moment will modify the cross section at low energies. There are further reasons to measure coherent neutrinonucleus scattering: neutrino-nucleus scattering processes are important in supernova physics [1] , as well as being useful themselves for supernova neutrino detection [3]. Because they are flavor blind, NC processes allow measurement of total neutrino flux, which can be compared to independently measured CC interactions. Therefore one can obtain limits on neutrino oscillation, and in particular on oscillations to sterile neutrinos [29]. Finally, it has even been proposed to exploit the large cross sections of neutrino-nucleus scattering for practical neutrino detectors, e.g. reactor monitoring [5, 6].

This section will discuss the various ways of probing new physics with a coherent elastic scattering experiment. At this stage, the experimental systematic uncertainty on the absolute rate is not known. It will depend on the specific detector type and configuration, backgrounds, and source uncertainties. However a total systematic uncertainty of $\sim 10 \%$ (including nuclear, beam and detector-related uncertainties), while perhaps optimistic, may well be achievable. Systematic uncertainties will likely dominate at the few tens of a kilogram scale or greater. 


\section{A. Weak Mixing Angle}

The SM predicts a coherent elastic scattering rate proportional to $Q_{w}^{2}$, the weak charge given by $Q_{w}=$ $N-\left(1-4 \sin ^{2} \theta_{W}\right) Z$, where $Z$ is the number of protons, $N$ is the number of neutrons and $\theta_{W}$ is the weak mixing angle. Therefore the weak mixing angle can be extracted from the measured absolute cross section, at a typical $Q$ value of $0.04 \mathrm{GeV} / c^{2}$. A deviation from the SM prediction could indicate new physics.

If the absolute cross section can be measured to $10 \%$, there will be an uncertainty on $\sin ^{2} \theta_{W}$ of $\sim$ $5 \%$. This is not competitive with the current best measurements from atomic parity violation [30, 31], SLAC E158 32] and NuTeV [33], which have better than percent-level uncertainties. One would need to significantly improve the systematic uncertainty on the absolute rate (perhaps by normalizing with a well-known rate) for coherent elastic $\nu \mathrm{A}$ scattering in order to make a useful measurement of the weak mixing angle. More promising would be a search for non-standard interactions of neutrinos with nuclei, as described in the following subsection.

\section{B. Non-Standard Interactions of Neutrinos}

Existing precision measurements of the weak mixing angle at low $Q$ do not constrain new physics which is specific to neutrino-nucleon interactions.

Here a model-independent parameterization of non-standard contributions to the cross section is used, following Refs. 34, 35]. In this description, one assumes an effective Lagrangian for interaction of a neutrino with a hadron:

$$
\begin{aligned}
& \mathcal{L}_{\nu H}^{N S} \underline{\underline{I}}-\frac{G_{F}}{\sqrt{2}} \sum_{\substack{q=u, d \\
\alpha, \beta=e, \mu, \tau}}\left[\bar{\nu}_{\alpha} \gamma^{\mu}\left(1-\gamma^{5}\right) \nu_{\beta}\right] \times \\
& \left(\varepsilon_{\alpha \beta}^{q L}\left[\bar{q} \gamma_{\mu}\left(1-\gamma^{5}\right) q\right]+\varepsilon_{\alpha \beta}^{q R}\left[\bar{q} \gamma_{\mu}\left(1+\gamma^{5}\right) q\right]\right) .
\end{aligned}
$$

The $\varepsilon$ parameters describe either "non-universal" $(\alpha=\beta)$ or flavor-changing $(\alpha \neq \beta)$ interactions.

As in Ref. 34], nuclei with total spin zero, and for which sum of proton spins and sum of neutron spins is also zero, are considered; in this case we have sensitivity to vector couplings, $\varepsilon_{\alpha \beta}^{q V}=\varepsilon_{\alpha \beta}^{q L}+\varepsilon_{\alpha \beta}^{q R}$. The cross section for coherent NC elastic scattering of neutrinos of flavor $\alpha$ off such a spin-zero nucleus is given by

$$
\begin{aligned}
& \left(\frac{d \sigma}{d E}\right)_{\nu_{\alpha} A}=\frac{G_{F}^{2} M}{\pi} F^{2}(2 M E)\left[1-\frac{M E}{2 k^{2}}\right] \times \\
& \left\{\left[Z\left(g_{V}^{p}+2 \varepsilon_{\alpha \alpha}^{u V}+\varepsilon_{\alpha \alpha}^{d V}\right)+N\left(g_{V}^{n}+\varepsilon_{\alpha \alpha}^{u V}+2 \varepsilon_{\alpha \alpha}^{d V}\right)\right]^{2}\right. \\
& \left.\quad+\sum_{\alpha \neq \beta}\left[Z\left(2 \varepsilon_{\alpha \beta}^{u V}+\varepsilon_{\alpha \beta}^{d V}\right)+N\left(\varepsilon_{\alpha \beta}^{u V}+2 \varepsilon_{\alpha \beta}^{d V}\right)\right]^{2}\right\}
\end{aligned}
$$

where $Z$ is the number of protons in the nucleus, $N$ is the number of neutrons, and $g_{V}^{p}=\left(\frac{1}{2}-2 \sin ^{2} \theta_{W}\right)$, $g_{V}^{n}=-\frac{1}{2}$ are the SM weak constants.

A stopped-pion neutrino source such as that at the SNS contains $\nu_{\mu}, \bar{\nu}_{\mu}$, and $\nu_{e}$. A coherent elastic $\nu \mathrm{A}$ scattering experiment employing such a source would therefore have sensitivity to all but $\varepsilon_{\tau \tau}$ couplings.

Existing constraints on the values of $\varepsilon_{\alpha \beta}^{P}(P=$ $L, R)$ are summarized in Ref. 35]. Table \selects those relevant for interactions of electron and muon flavor neutrinos with quarks. New constraints from existing and future atmospheric, beam and solar neutrino experiments are explored in Refs. [36, 37].

TABLE I: Constraints on NSI parameters, from Ref. [35].

\begin{tabular}{cc}
\hline \hline NSI Parameter Limit & Source \\
\hline$-1<\varepsilon_{e e}^{u L}<0.3$ & CHARM $\nu_{e} N, \bar{\nu}_{e} N$ scattering \\
$-0.4<\varepsilon_{e e}^{u R}<0.7$ & \\
$-0.3<\varepsilon_{e e}^{d L}<0.3$ & CHARM $\nu_{e} N, \bar{\nu}_{e} N$ scattering \\
$-0.6<\varepsilon_{e e}^{d R}<0.5$ & \\
$\left|\varepsilon_{\mu \mu}^{u L}\right|<0.003$ & NuTeV $\nu N, \bar{\nu} N$ scattering \\
$-0.008<\varepsilon_{\mu \mu}^{u R}<0.003$ & \\
$\left|\varepsilon_{\mu \mu}^{d L}\right|<0.003$ & NuTeV $\nu N, \bar{\nu} N$ scattering \\
$-0.008<\varepsilon_{\mu \mu}^{d R}<0.015$ & \\
$\left|\varepsilon_{e \mu}^{u P}\right|<7.7 \times 10^{-4}$ & $\mu \rightarrow e$ conversion on nuclei \\
$\left|\varepsilon_{e \mu}^{d P}\right|<7.7 \times 10^{-4}$ & $\mu \rightarrow e$ conversion on nuclei \\
$\left|\varepsilon_{e \tau}^{u P}\right|<0.5$ & $\mathrm{CHARM} \nu_{e} N, \bar{\nu}_{e} N$ scattering \\
$\left|\varepsilon_{e \tau}^{d P}\right|<0.5$ & $\mathrm{CHARM} \nu_{e} N, \bar{\nu}_{e} N$ scattering \\
$\left|\varepsilon_{\mu \tau}^{u P}\right|<0.05$ & $\mathrm{NuTeV} \nu N, \bar{\nu} N$ scattering \\
$\left|\varepsilon_{\mu \tau}^{d P}\right|<0.05$ & $\mathrm{NuTeV} \nu N, \bar{\nu} N$ scattering \\
\hline \hline
\end{tabular}

From this table, one can see that of these parameters, $\varepsilon_{e e}$ and $\varepsilon_{e \tau}$ are quite poorly constrained: values of order unity are allowed. $\left|\varepsilon_{\mu \beta}\right|$ couplings are, however, constrained to better than 0.05. Given this situation, the focus here is on $\varepsilon_{e e}$ and $\varepsilon_{e \tau}$ couplings [38]. These would be accessible using the electron flavor component of the source. That no oscillations take place (i.e. that the standard three-flavor model of neutrino mixing holds, and that the baseline is too short for significant flavor transition) is also assumed.

The signature of NSI is a deviation from the expected cross section. The following show a few ex- 
amples of two-dimensional slices of regions in $\varepsilon_{\alpha \beta}$ parameter space that would be allowed if one measured exactly the SM expectation.

Fig. [7] shows $90 \%$ C. L. allowed regions one would draw for $\varepsilon_{e e}^{u V}, \varepsilon_{e e}^{d V}$, if the rate predicted by the SM were measured for the delayed flux (which contains $\nu_{e}$ ), assuming that the $\varepsilon_{\mu \beta}$ parameters are negligible, and for $\varepsilon_{e \tau}^{q V}=0$, for $100 \mathrm{~kg}$-yr of running of a neon detector at $20 \mathrm{~m}$ from the source. A $10 \mathrm{keV}$ threshold is assumed. This calculation considers only the total delayed $\left(\nu_{e}+\bar{\nu}_{\mu}\right)$ flux rate [39]. The regions corresponding to assumptions of $5 \%$ and $10 \%$ systematic error in addition to statistical error, and for statistical error alone are shown [40]. As before, a perfectly efficient, background-free detector is assumed.

Note that in Eq. 4 even in the presence of nonuniversal NSI, one can obtain rates identical to the $\mathrm{SM}$ prediction in the case that

$$
\begin{array}{r}
Z\left(g_{V}^{p}+2 \varepsilon_{e e}^{u V}+\varepsilon_{e e}^{d V}\right)+N\left(g_{V}^{n}+\varepsilon_{e e}^{u V}+2 \varepsilon_{e e}^{d V}\right) \\
= \pm\left(Z g_{V}^{p}+N g_{V}^{n}\right),
\end{array}
$$

so for

$$
\varepsilon_{e e}^{u V}=-\frac{(A+N)}{(A+Z)} \varepsilon_{e e}^{d V}
$$

and

$$
\varepsilon_{e e}^{u V}=-\frac{(A+N)}{(A+Z)} \varepsilon_{e e}^{d V}-\frac{2\left(Z g_{V}^{p}+N g_{V}^{n}\right)}{A+Z} .
$$

For this reason, allowed regions of Fig. [ 7 appear as linear bands in $\varepsilon_{e e}^{u V}, \varepsilon_{e e}^{d V}$ parameter space. A measurement employing more than one element can then place more stringent constraints on the couplings; the more the $(A+N) /(A+Z)$ ratio differs between the two targets, the better.

Fig. 8 shows the same regions for $100 \mathrm{~kg}$-yr each of ${ }^{132} \mathrm{Xe}$ and ${ }^{20} \mathrm{Ne}$, where the black ellipses represent the $90 \%$ allowed region from the combination of the measurements. These regions are superposed on the allowed region from high-energy $\nu_{e}$ scattering on nucleons derived from CHARM experiment results 41] in Ref. [35], for the case that the axial parameters $\varepsilon_{e e}^{q A}=\varepsilon_{e e}^{q L}-\varepsilon_{e e}^{q R}$ are zero.

Fig. [9] shows similar $90 \%$ allowed regions for a slice of $\varepsilon_{e e}^{d V}, \varepsilon_{e \tau}^{d V}$ parameter space, for $\varepsilon_{e e}^{u V}=\varepsilon_{e \tau}^{u V}=0$ (note that $d$-quark NSI may be especially interesting; see e.g. [42]). In this case the allowed parameters correspond to regions between two ellipses. Fig. 9] shows regions for a ${ }^{20} \mathrm{Ne}$ detector (with same assumptions as above). Fig. 10 shows the result for ${ }^{132} \mathrm{Xe}$ as well, and the black ellipses contain the region allowed by the combined measurements (for this case, only a small improvement is afforded by measurements with multiple targets).

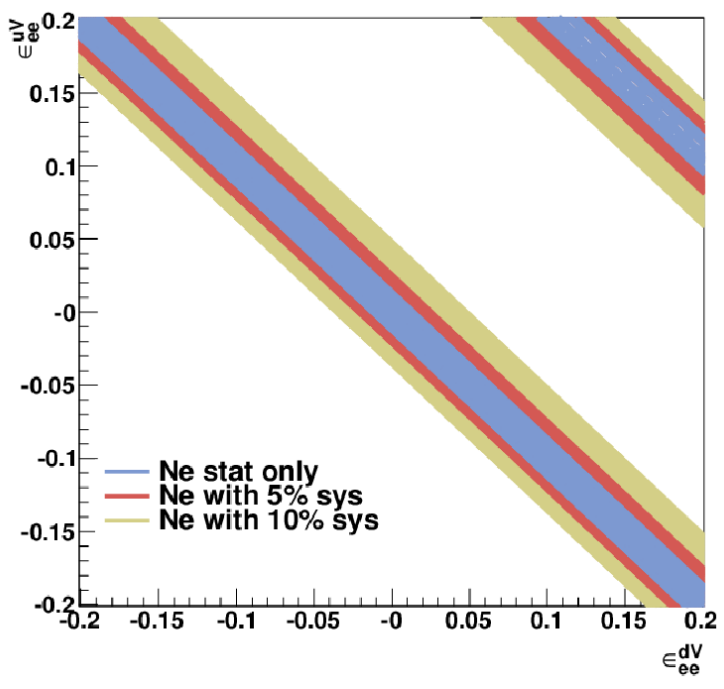

FIG. 7: Allowed region at $90 \%$ C.L. for $\varepsilon_{e e}^{u V}$ and $\varepsilon_{e e}^{d V}$, for $100 \mathrm{~kg}-\mathrm{yr}$ of ${ }^{20} \mathrm{Ne}$ at the SNS. The outer region corresponds to an assumed systematic uncertainty of $10 \%$ in addition to statistical uncertainty; the middle region corresponds to an assumed systematic uncertainty of $5 \%$, and the inner region corresponds to statistical uncertainty only.

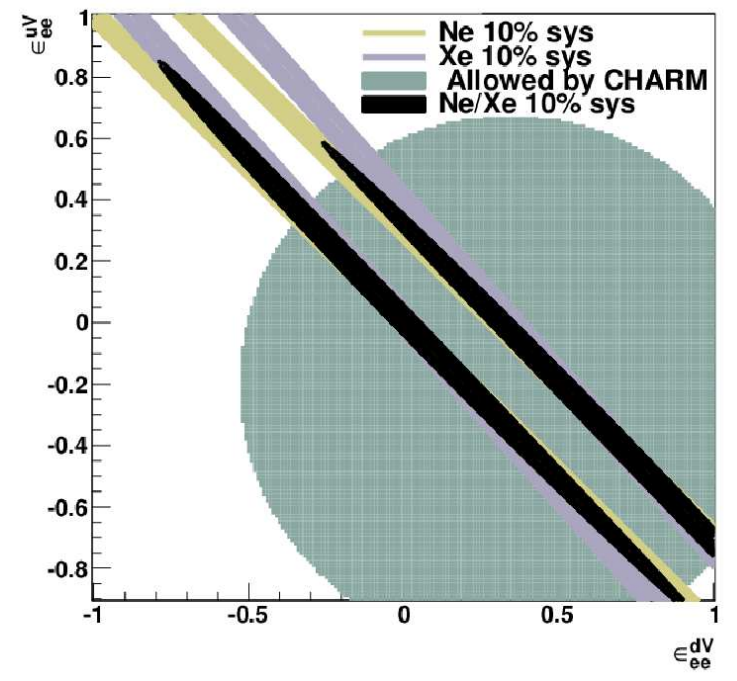

FIG. 8: Allowed regions at $90 \%$ C.L. for $\varepsilon_{e e}^{u V}$ and $\varepsilon_{e e}^{d V}$, for $100 \mathrm{~kg}$-yr each of ${ }^{20} \mathrm{Ne}$ and ${ }^{132} \mathrm{Xe}$ (steeper slope band) at the SNS, assuming $10 \%$ systematic uncertainty, plus statistical uncertainty. The thin black ellipses correspond to combined Ne/Xe measurement. The shaded elliptical region corresponds to a slice of the CHARM experiment's allowed NSI parameter space, for $\varepsilon_{e e}^{q A}=0$.

Fig. 11] compares neutrino-nucleus scattering sensitivity to allowed NSI parameters derived based on lack of distortion of oscillation parameters for beam 


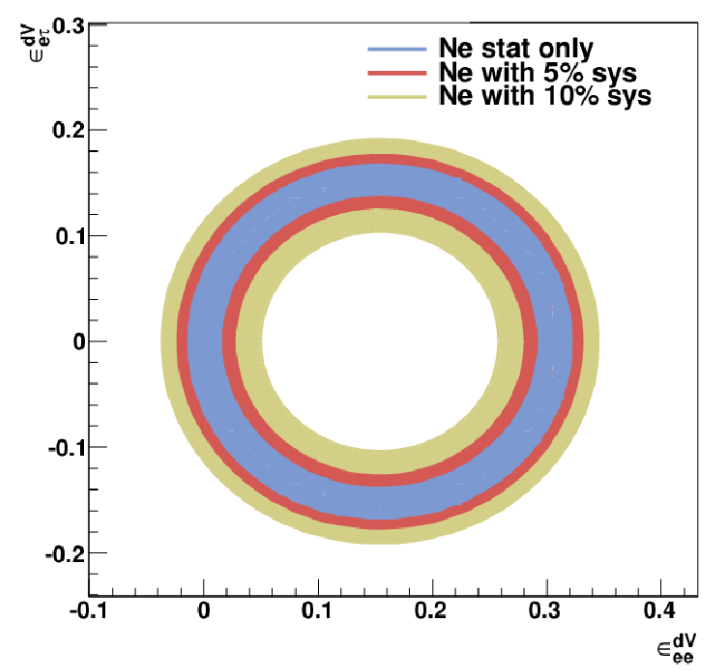

FIG. 9: Allowed region at $90 \%$ C.L. for $\varepsilon_{e e}^{d V}$ and $\varepsilon_{e \tau}^{d V}$, for $100 \mathrm{~kg}-\mathrm{yr}$ of ${ }^{20} \mathrm{Ne}$ at the SNS. The shaded region between the outer and inner ellipses corresponds to an assumed systematic uncertainty of $10 \%$ in addition to statistical uncertainty; the next largest region corresponds to an assumed systematic uncertainty of $5 \%$, and the inner region corresponds to statistical uncertainty only.

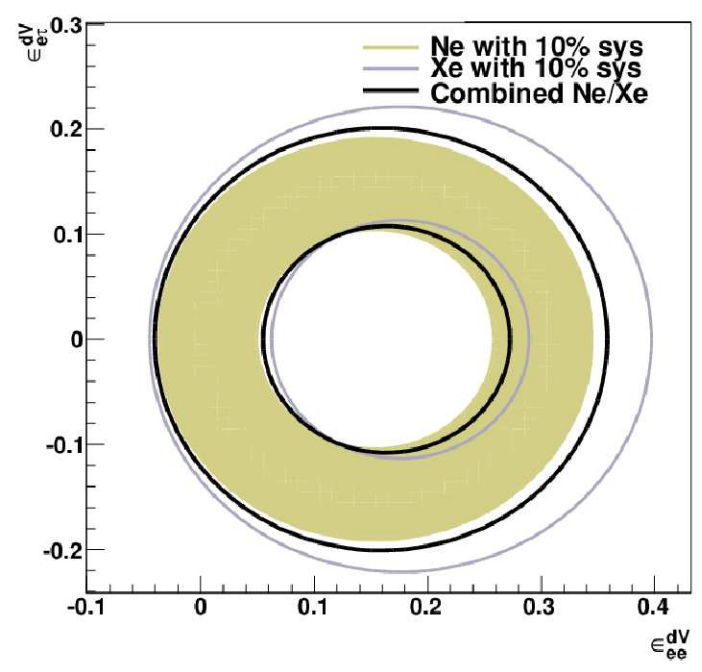

FIG. 10: Allowed regions with same assumptions as Fig. 9] $10 \%$ systematic uncertainty, for ${ }^{20} \mathrm{Ne}$ (shaded region), ${ }^{132} \mathrm{Xe}$ (region between pale lines) and both combined (region between black lines).

and atmospheric neutrinos 36 .

It is worth noting that a coherent neutrino-nucleus elastic scattering experiment will provide significant constraints on still-allowed NSI parameters that modify solar neutrino survival probabilities [37]. As a case in point, consider specific NSI parameters

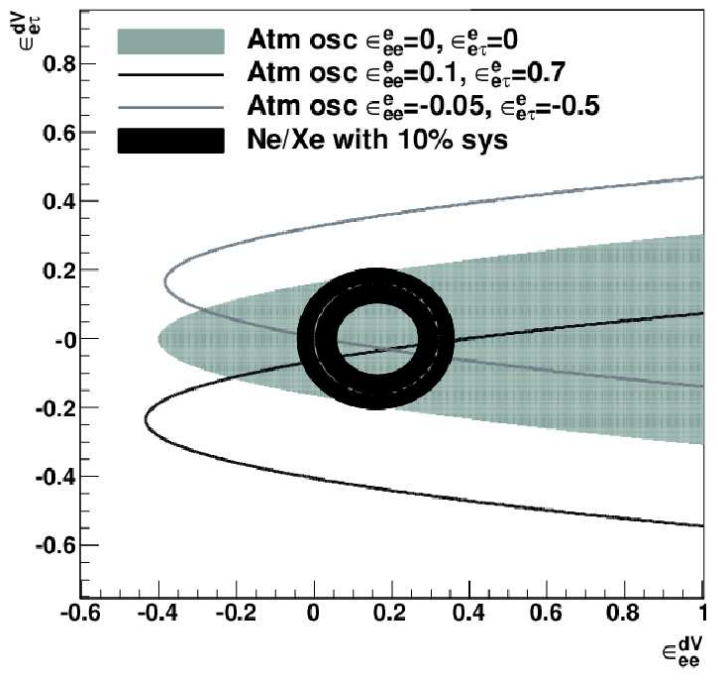

FIG. 11: Allowed region at 90\% C.L. for $\varepsilon_{e e}^{d V}$ and $\varepsilon_{e \tau}^{d V}$, $\varepsilon_{e e}^{u V}=\varepsilon_{e \tau}^{u V}=0$, for $100 \mathrm{~kg}-\mathrm{yr}$ each of ${ }^{20} \mathrm{Ne}$ and ${ }^{132} \mathrm{Xe}$ at the SNS is shown in black. The shaded region corresponds to a slice of allowed NSI parameters from Ref. [36] for $\varepsilon_{\tau \tau}=0$, and $\varepsilon_{e e}^{e V}=\varepsilon_{e \tau}^{e V}=0$; the parabolic regions inside the dark and light lines correspond to slices of allowed parameter space for some specific values of $\varepsilon_{e e}^{e V}$ and $\varepsilon_{e \tau}^{e V}$.

that yield the "LMA-0" solution of Ref. [37]: $\varepsilon_{11}^{u V}=$ $\varepsilon_{11}^{d V}=-0.065$, and $\varepsilon_{12}^{u V}=\varepsilon_{12}^{d V}=-0.15$, where $\varepsilon_{11}=\varepsilon_{e e}-\varepsilon_{\tau \tau} \sin ^{2} \theta_{23}$, and $\varepsilon_{12}=-2 \varepsilon_{e \tau} \sin \theta_{23}$, and $\theta_{23}$ is the atmospheric mixing angle, known to be $\sim \pi / 4$. Following the approach in this reference, $\varepsilon_{\alpha \beta}^{u V}=\varepsilon_{\alpha \beta}^{d V}$ is assumed. Fixing $\varepsilon_{11}$ and $\varepsilon_{12}$ defined in this way will yield different neutrinonucleus scattering constraints for different assumptions of $\varepsilon_{\tau \tau}$. Fig. 12] shows the value of a $\chi^{2}$ defined as $\chi^{2}=\left(N_{\text {NSI }}-N_{S M}\right)^{2} / \sigma^{2}$, as a function of $\varepsilon_{\tau \tau}^{u V}=\varepsilon_{\tau \tau}^{d V} ; N_{\text {NSI }}$ is the number of signal events for the given NSI parameters, and $N_{\mathrm{SM}}$ is the SM expectation, for $100 \mathrm{~kg}-\mathrm{yr}$ of ${ }^{20} \mathrm{Ne}$ at $10 \mathrm{keV}$ threshold; $\sigma^{2}$ includes both statistical uncertainty and an assumed $10 \%$ systematic uncertainty. Superimposed on this plot as a shaded region is the restriction on $\varepsilon_{\tau \tau}^{(u, d) V}$ (given assumptions above, and $\varepsilon_{\alpha \beta}^{e V}=0$ ) from beam and atmospheric neutrino oscillations, $\left|1+\varepsilon_{e e}+\varepsilon_{\tau \tau}-\sqrt{\left(1+\varepsilon_{e e}-\varepsilon_{\tau \tau}\right)^{2}+4\left|\varepsilon_{e \tau}\right|^{2}}\right|<0.4$, taken from Ref. [36].

From this plot one can see that a coherent elastic neutrino-nucleus scattering experiment has sensitivity to the set of NSI parameters for which the LMA0 region is derived. If the rate predicted by the SM were to be observed, these LMA-0 parameters could be ruled out for $\varepsilon_{\tau \tau}=0$, and would remain viable only for a restricted range of $\varepsilon_{\tau \tau}^{(u, d) V}$. 


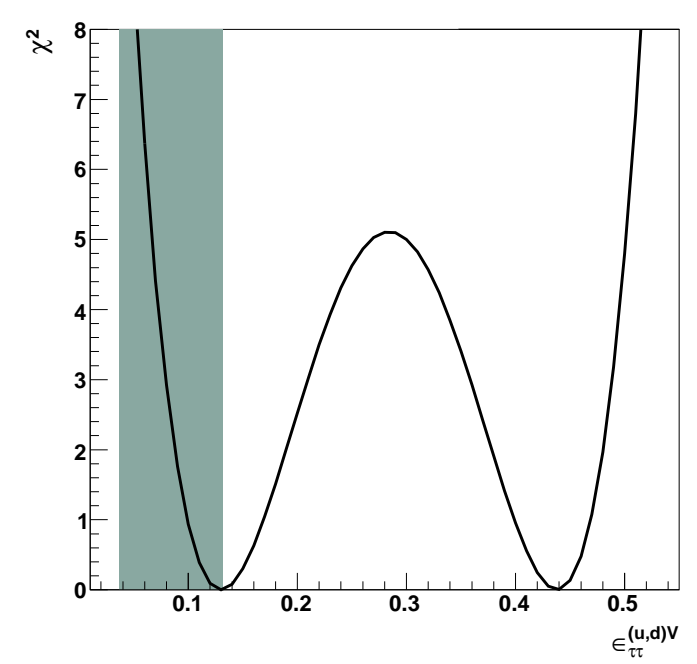

FIG. 12: $\chi^{2}$ as a function of $\varepsilon_{\tau \tau}^{(u, d) V}$ for $100 \mathrm{~kg}$-yrs of ${ }^{20} \mathrm{Ne}$, assuming NSI parameters $\varepsilon_{11}^{(u, d) V}=-0.065$ and $\varepsilon_{12}^{(u, d) V}=-0.15$. The shaded region represents allowed $\varepsilon_{\tau \tau}$ parameters from Ref. [36], from beam and atmospheric neutrino oscillation constraints.

The conclusion of these NSI studies is that a coherent elastic neutrino-nucleus scattering experiment at a stopped-pion source would have significant sensitivity to currently-allowed NSI $\varepsilon_{e e}^{q V}$ and $\varepsilon_{e \tau}^{q V}$ parameters.

\section{Neutrino Magnetic Moment}

The SM predicts a neutrino magnetic moment of $\mu_{\nu} \leq 10^{-19} \mu_{B}\left(m_{\nu} / 1 \mathrm{eV}\right)$, in units of Bohr magnetons. This is very small, but extensions of the SM commonly predict larger ones. The most stringent limits are astrophysical: for instance, based on lack of observed energy loss from electromagnetic couplings in red giant evolution one can set a limit $\mu_{\nu} \leq 10^{-12} \mu_{B}$ [43. The best direct experimental limits result from lack of distortion of neutrinoelectron elastic scattering at low energy, and are in the range of $\mu_{\nu}\left(\nu_{e}\right) \leq 1-2 \times 10^{-10} \mu_{B}$ [44, 45, 46]. For muon neutrino scattering, the best limit is less stringent: $\mu_{\nu}\left(\nu_{\mu}\right) \leq 6.8 \times 10^{-10} \mu_{B}$ [47].

A signature of non-zero neutrino magnetic moment can be observed via distortion of the recoil spectrum of coherently scattered nuclei. The magnetic scattering cross section is given in Ref. [48] for a spin-zero nucleus:

$$
\left(\frac{d \sigma}{d E}\right)_{m}=\frac{\pi \alpha^{2} \mu_{\nu}^{2} Z^{2}}{m_{e}^{2}}\left(\frac{1-E / k}{E}+\frac{E}{4 k^{2}}\right)
$$

Fig. 13 shows the differential cross sections calculated for ${ }^{20} \mathrm{Ne}$, for $30 \mathrm{MeV}$ neutrino energy, as a function of nuclear recoil energy. The magnetic scattering cross section is calculated for neutrino magnetic moment just below the current best experimental limits $\left(10^{-10} \mu_{B}\right.$ for $\nu_{e}$ and $6 \times 10^{-10} \mu_{B}$ for $\left.\nu_{\mu}\right)$.

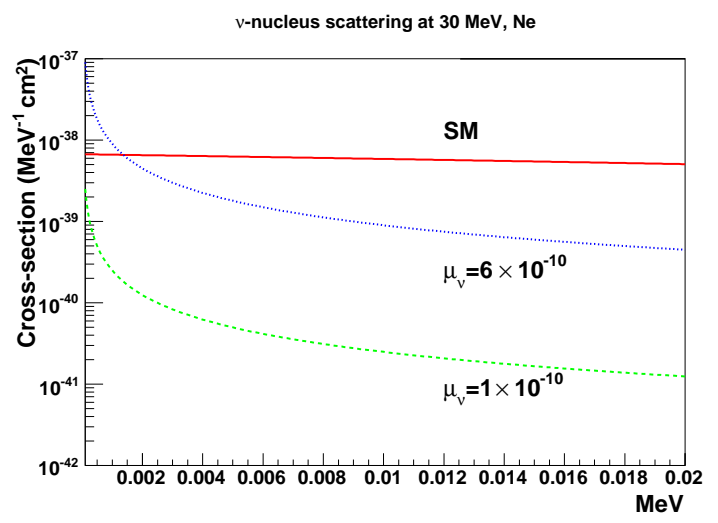

FIG. 13: Solid line: SM coherent neutrino-nucleus differential cross section, as a function of nuclear recoil energy $E$, for neutrino energy $k=30 \mathrm{MeV}$ and for a ${ }^{20} \mathrm{Ne}$ target. Dashed line: differential cross section for neutrinonucleus scattering due to a neutrino magnetic moment of $\mu_{\nu}=10^{-10} \mu_{B}$. Dotted line: differential cross section for neutrino-nucleus scattering due to a neutrino magnetic moment of $\mu_{\nu}=6 \times 10^{-10} \mu_{B}$.

Fig. 14] shows the yield in events per keV of recoil energy, per ton per year in a neon detector at $20 \mathrm{~m}$ from the SNS target, with and without neutrino magnetic moment contribution, for prompt and delayed fluxes. The dashed line assumes $\nu_{\mu}=10^{-10} \mu_{B}$ for both $\nu_{e}$ and $\bar{\nu}_{\mu}$. The dotted line assumes $\nu_{\mu}=$ $10^{-10} \mu_{B}$ for $\nu_{e}$ and $\nu_{\mu}=6 \times 10^{-10} \mu_{B}$ for $\bar{\nu}_{\mu}$.

The difference in coherent neutrino-nucleus scattering yield due to presence of a neutrino magnetic moment near the current $\mu_{\nu}$ limit for $\nu_{e}$ is very small, except for recoil energies below a few $\mathrm{keV}$. This signal is therefore likely out of reach for a CLEAN-type experiment at the SNS. However, for $\mu_{\nu}$ near the current limit for $\nu_{\mu}$, there might be a measurable signal for a $10 \mathrm{keV}$ threshold, and it is conceivable that one could improve the limit with a high-statistics measurement. Nuclei with spin, although not considered here, have additional $\mu_{\nu}$-dependent terms in their coherent neutrino-nucleus scattering cross sections [48] and may be potential targets for a neutrino magnetic moment search [4] 

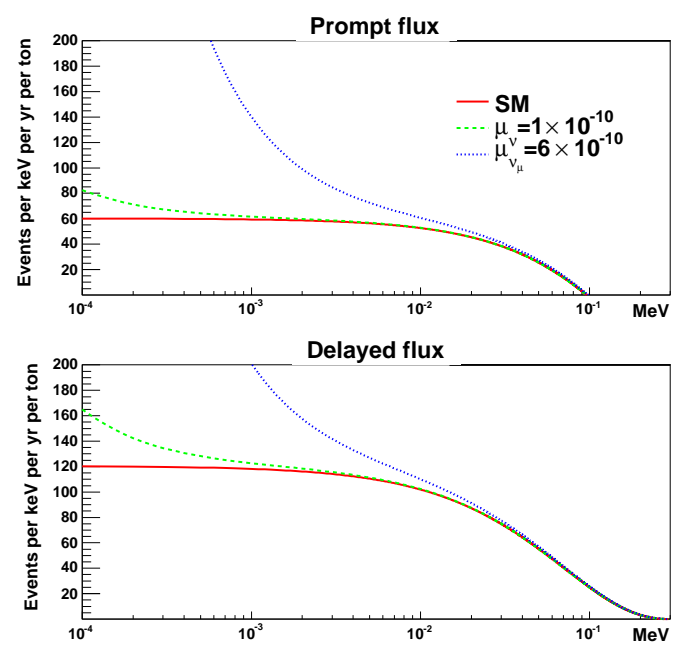

FIG. 14: Differential yield at the SNS in neon as a function of nuclear recoil energy. The top plot is for the prompt flux $\left(\nu_{\mu}\right.$ only) and the bottom plot is for the delayed flux (sum of $\nu_{e}$ and $\bar{\nu}_{\mu}$ ). Solid lines: SM expectation. Dashed lines: yield including magnetic moment contribution for $\mu_{\nu}=10^{-10} \mu_{B}$ for both $\nu_{e}$ and $\bar{\nu}_{\mu}$. Dotted lines: yield including magnetic moment contribution for $\mu_{\nu}=10^{-10} \mu_{B}$ for $\nu_{e}$ and $\mu_{\nu}=6 \times 10^{-10} \mu_{B}$ for $\bar{\nu}_{\mu}$, $\nu_{\mu}$.

\section{CONCLUSION}

Straightforward calculations indicate that one expects thousands of coherent neutrino-nucleus interactions with recoil energies $>10 \mathrm{keV}$ per ton of material per year of running at the SNS, which is very promising. Even few kilogram-scale experiments may have measurable rates. These estimates have been made for an experiment with no background and no inefficiency; both will certainly be important for a real experiment. Sensitivities will need to be reevaluated for a specific detector configuration for which backgrounds and efficiencies can be estimated.

Unambiguous detection of the process is a first step; high statistics measurements will then follow. Such an experiment has significant potential for constraining NSI parameters; magnetic moment and precision weak mixing angle measurements are also conceivable, although pose a greater experimental challenge.

\section{Acknowledgments}

The author is grateful to D. Akerib, B. Balantekin, J. Beacom, J. Collar, M. Dragowsky, Y. Efremenko, A. Friedland, H. Gao, C. Horowitz, E. Kearns, G. McLaughlin, B. Mueller, R. Raghavan, T. Schutt, C. Walter and A. Young, and especially J. Engel, C. Lunardini and D. McKinsey, for comments and discussions. C. Horowitz provided the form factors for the calculation. The author would also like to thank the Institute for Nuclear Theory at the University of Washington, where this work was started, for its hospitality. The author's research activities are supported by the Department of Energy and the National Science Foundation.
[1] D. Z. Freedman, D. N. Schramm, and D. L. Tubbs, Ann. Rev. Nucl. Part. Sci. 27, 167 (1977).

[2] A. Drukier and L. Stodolsky, Phys. Rev. D30, 2295 (1984).

[3] C. J. Horowitz, K. J. Coakley, and D. N. McKinsey, Phys. Rev. D68, 023005 (2003), astro-ph/0302071.

[4] F. Boehm and P. Vogel, Physics of Massive Neutrinos (Cambridge University Press, Cambridge, 1987).

[5] P. Barbeau, J. I. Collar, J. Miyamoto, and I. Shipsey, IEEE Trans. Nucl. Sci. 50, 1285 (2003), hep-ex/0212034.

[6] C. Hagmann and A. Bernstein, IEEE Trans. Nucl. Sci. 51, 2151 (2004), nucl-ex/0411004.

[7] H. T. Wong (2005), hep-ex/0511001.

[8] D. N. McKinsey and K. J. Coakley, Astropart. Phys. 22, 355 (2005), astro-ph/0402007.

[9] M. G. Boulay, A. Hime, and J. Lidgard (2004), nuclex/0410025.

[10] M. G. Boulay and A. Hime (2004), astro- $\mathrm{ph} / 0411358$.

[11] E. Aprile et al., Phys. Rev. D72, 072006 (2005), astro-ph/0503621.

[12] Y. Takeuchi, in Proceedings of the 32nd International Conference on High-Energy Physics (ICHEP 04), Beijing, China, 16-22 Aug 2004 (World Scientific, Hackensack, 2004).

[13] G. J. Alner et al. (UK Dark Matter), Astropart. Phys. 23, 444 (2005).

[14] D. P. Snowden-Ifft, T. Lawson, N. J. C. Spooner, and N. Villaume, Nucl. Instrum. Meth. A516, 406 (2004).

[15] R. Galea (2005), http://snolab2005.snolab.ca/talks/ Snolab_workshopIV_galea_ebubble.pdf.

[16] D. S. Akerib et al., Nucl. Instrum. Meth. A520, 163 (2004).

[17] D. S. Akerib et al., Nucl. Instrum. Meth. A520, 116 (2004).

[18] W. J. Bolte et al. (2005), astro-ph/0503398.

[19] M. Barnabe-Heider et al. (PICASSO), Nucl. In- 
strum. Meth. A555, 184 (2005), physics/0508098.

[20] C. Athanassopoulos et al. (LSND), Nucl. Instrum. Meth. A388, 149 (1997), nucl-ex/9605002.

[21] R. L. Burman, Nucl. Instrum. Meth. A368, 416 (1996).

[22] I. S. K. Gardner, in 6th European Particle Accelerator Conference (EPAC 98), Stockholm, Sweden, 2226 Jun 1998 (IOP Publishing, Philadelphia, 1998).

[23] Y. Ikeda (2003), http://www.fz-juelich.de/ess/ datapool/icanspdf/Ikeda-G3-paper.pdf.

[24] F. T. Avignone and Y. V. Efremenko, J. Phys. G29, 2615 (2003).

[25] Nu-SNS Collaboration http://www.phy.ornl.gov/nusns/proposal.pdf.

[26] C. J. Horowitz and G. Shen (2005), private communication.

[27] C. J. Horowitz (2005), private communication.

[28] L. M. Krauss, Phys. Lett. B269, 407 (1991).

[29] Note that for an SNS configuration, most sterile oscillation scenarios are already quite well constrained by other experiments, especially assuming a null result from Mini-BooNE.

[30] S. C. Bennett and C. E. Wieman, Phys. Rev. Lett. 82, 2484 (1999), hep-ex/9903022.

[31] S. Eidelman et al. (Particle Data Group), Phys. Lett. B592, 1 (2004).

[32] P. L. Anthony et al. (SLAC E158), Phys. Rev. Lett. 95, 081601 (2005), hep-ex/0504049.

[33] G. P. Zeller et al. (NuTeV), Phys. Rev. Lett. 88, 091802 (2002), hep-ex/0110059.

[34] J. Barranco, O. G. Miranda, and T. I. Rashba, JHEP 12, 021 (2005), hep-ph/0508299.

[35] S. Davidson, C. Peña Garay, N. Rius, and A. Santamaria, JHEP 03, 011 (2003), hep-ph/0302093.

[36] A. Friedland and C. Lunardini, Phys. Rev. D72, 053009 (2005), hep-ph/0506143.

[37] A. Friedland, C. Lunardini, and C. Peña Garay,
Phys. Lett. B594, 347 (2004), hep-ph/0402266.

[38] One might conceivably eventually confront the current best $\varepsilon_{\mu \tau}$ limits with an SNS coherent elastic scattering experiment.

[39] In principle one might do better with a spectral fit to separate $\nu_{\mu}$ and $\bar{\nu}_{e}$ contributions.

[40] Note that one might achieve some cancellation of absolute source-flux-related systematic uncertainty by taking a ratio of prompt and delayed fluxes.

[41] J. Dorenbosch et al. (CHARM), Phys. Lett. B180, 303 (1986).

[42] P. S. Amanik, G. M. Fuller, and B. Grinstein, Astropart. Phys. 24, 160 (2005), hep-ph/0407130.

[43] G. G. Raffelt, Phys. Rept. 320, 319 (1999).

[44] D. W. Liu et al. (Super-Kamiokande), Phys. Rev. Lett. 93, 021802 (2004), hep-ex/0402015.

[45] Z. Daraktchieva et al. (MUNU), Phys. Lett. B615, 153 (2005), hep-ex/0502037.

[46] H. T. Wong, Nucl. Phys. Proc. Suppl. 143, 205 (2005), hep-ex/0409003.

[47] L. B. Auerbach et al. (LSND), Phys. Rev. D63, 112001 (2001), hep-ex/0101039.

[48] P. Vogel and J. Engel, Phys. Rev. D39, 3378 (1989).

[49] Note that a neutrino-electron magnetic scattering search can in principle be done at the SNS with low threshold detectors. The $\nu$ magnetic scattering cross-section is smaller by a factor of $Z^{2}$ than for neutrino-nucleus, but there are a factor of $Z$ more electron targets, so the signal is overall a factor of 10 smaller than for $\nu A$. This amounts to $\sim 10 \nu e$ magnetic scattering events per ton per year at the SNS above $10 \mathrm{keV}$, for $\mu_{\nu}$ at the current $\nu_{\mu}$ limit. The $\nu e$ magnetic signal has negligible SM background; however absolute rates are low for realistic target masses and likely to be subject to experimental backgrounds. 